\title{
The Effect of a Selective Pilates Program on Functional Balance and Falling Risk in Patients with Parkinson's Disease
}

\author{
Hasan Daneshmandi, ${ }^{1}$ Shaghayegh Sayyar, ${ }^{1, *}$ and Babak Bakhshayesh ${ }^{2}$ \\ ${ }^{1}$ Department of Sport Injuries and Corrective Exercises, Faculty of Physical Education and Sport Sciences, University of Guilan, Rasht, Iran \\ ${ }^{2}$ Department of Neurology, Guilan University of Medical Sciences, Rasht, Iran \\ "Corresponding author: Shaghayegh Sayyar, Department of Sport Injuries and Corrective Exercises, Faculty of Physical Education and Sport Sciences, University of Guilan, \\ Rasht, Iran. E-mail: shaghayegh.sayyar@yahoo.com
}

Received 2016 July 13; Revised 2016 December 07; Accepted 2017 April 22.

\begin{abstract}
Background: Parkinsonism is a progressive disorder of the nervous system for which only symptomatic treatment exists. Balance dysfunction and postural instability observed in persons with Parkinson's disease are important components of the disability. Objectives: To investigate the effect of a selective 8-week Pilates exercises on the functional balance and falling risk in patients with idiopathic Parkinson's disease.

Methods: This randomized controlled trial included 30 patients with idiopathic Parkinson's disease who were selected as subjects non-randomly and purposely, and assigned into exercise groups or control group. Participants in exercise and control groups attended an 8-week Pilate's program or walking program respectively. Functional balance and falling risk was evaluated using Fullerton balance scale (FAB) and timed up and go test (TUG), before and after interventions. In order to analyze the data paired samples T-test and independent samples T-test and SPSS version 22 were used.

Results: Attending an eight-week Pilates exercise was associated with a significant improvement in functional balance and falling risk $(\mathrm{P}=0.001)$, while the difference of the functional balance and falling risk, before and after performing the protocol, in control group was not significant $(\mathrm{P}=0.364)$. Also the difference between the results of functional balance and falling risk scale scores of two groups in post-test were significant $(\mathrm{P}=0.001)$.

Conclusions: Given that Pilate's exercises involve both musculoskeletal system and nervous system, can be an effective intervention to improving balance and reducing falling risk, especially in people with postural control disabilities.
\end{abstract}

Keywords: Parkinson's Disease, Exercise, Balance, Falling Risk

\section{Background}

Parkinson's disease (PD) is a debilitating chronic neurodegenerative disorder affecting the dopamineproducing cells in the substantia nigra within the basal ganglia resulting in motor dysfunction, which leads to weakness, pain, and tightness, difficulty in walking, rising from chairs, clumsy movements and a decline in physical activity. It is the second most common neurological disease in the world that affects neurophysiologic function, movement abilities, and quality of life (QOL) [1,2]. Tremor, bradykinesia, rigidity, balance dysfunction and postural instability are the common incapacitating symptoms of PD. Untreated balance dysfunction and postural instability and gait difficulty are highly associated with falls in this population which in turn increases the chance of developing comorbidity and disability by causing alterations in postural control strategies during standing tasks and when performing voluntary movements. Balance dysfunction and postural instability are also associated with a loss of equilibrium, sudden falls, progressive loss of independence and immobility [1-5]. Among people with
$\mathrm{PD}$, as many as $65 \%$ of fallers will experience an injury secondary to their falls, 33\% will suffer a fracture and $75 \%$ of falls will lead to use of a health care service. This results in restriction of activities, compromising quality of life and predisposing to secondary reductions in muscle strength and cardiovascular fitness [6].

Regular physical exercises appear to afford therapeutic benefits, increasing mobility and quality of life, in some people living with PD [7]. The growth and popularity of complementary physical therapies for PD attempt to fill the gap left by exercise interventions, which does not always directly target wellbeing, enjoyment and social participation $[1,7,8]$. There is mounting evidence that exercise is beneficial for PD [9-11]. Exercise is an integral part of the management of PD, because physical activity has been shown to retard the deterioration of motor functions and to prolong functional independence. Resistance-based exercises that address deficits in balance and strength have shown positive effects. However, they require safety monitoring and are equipment-dependent. Research on alternative forms of exercise that could improve balance, gait, 
and function in patients with PD is scarce [12-15]. On balance, alternative physical therapies are worthy of consideration when selecting treatment options for people with this common chronic disease [7].

Pilates has become a popular exercise modality that combines strength and flexibility training and, with the proposed benefits of improved muscular control of the deeper abdominal muscles, may provide an effective method of improving postural stability [16]. Pilates aims to improve co-ordination and control of the core muscles of the trunk, which contribute to the optimal lumbar-pelvic stabilization needed for daily activities and function [17]. Pilates is considered appropriate for all ages and fitness status and has been used in multiple conditions, such as aged related disabilities [18]. Previous studied demonstrated that Pilates improved balance and reduced the number of falls in older adults $[17,19,20]$. However, there is a lack of studies with high methodological quality regarding the effects of Pilates especially in people with neurodegenerative diseases. Pilates has been shown to improve balance in elderly individuals, but its impact on balance and falling risk in idiopathic Parkinson's disease (IPD) has not been evaluated. Therefore the current study was planned to assess the effects of an eight-week Pilates exercise program on balance and risk of falling in patients with IPD.

\section{Methods}

The present research is quasi-experimental, applied and randomized clinical trial in which the quasiexperimental pretest-posttest design was used with a control group. The research statistical population includes all patients with PD who referred to neurologists in 2016 in Guilan Province. After the necessary coordination, all patients with Parkinson's disease were examined by neurologists in terms of eligibility for participating in the research in order to select subjects. From among the desired people, 32 patients with IPD were selected through available targeted non-random method considering the entry and exit criteria and given the person's satisfaction and they were randomly and consecutively divided into two experimental and control groups. Referring to similar studies and considering the sample size required for experimental causal researches, the minimum size of each group is 15 people. On the other hand, considering the possibility of subjects' withdrawal for any reason and for preventing from disruption due to the possible loss of subjects, the number of subjects was considered 16 people for each group. It is worth mentioning that during the research procedure, one person per group stopped continuing the research due to side effects of physical activity (such as fatigue, muscle pain, cramp, and so on) and finally the number of groups' subjects reduced to 15 people. The research entry criteria include having IPD at moderate levels (levels II and III), age over 50 years old, passage of at least 3 years after the disease diagnosis, lack of other neurological diseases or any acute and chronic physical or mental disorder, lack of cognitive disorders with the approval of a specialist physician, the ability to stand and walk independently, not using assistive devices for keeping balance and walking, the history of balancing and walking disorders due to PD, non-participation in sports activities and or physiotherapy treatments influencing the research process, and the ability to participate in practice sessions regularly. The exit criteria include pain, limbs' deformation and any disorder influencing the security of practice program and or unwillingness to be present at the research process. Before starting the research project, during a briefing session, all subjects got familiar with the environment of research and the method of study, and any ambiguity was removed and any question was answered. Considering the physical characteristics of patients with PD and age group of these people, the environment of research was completely immunized and selected near a treatment center in order to have access to treatment measures in the shortest possible time in case of occurrence of any possible problem. Then, all participants in this study completed the consent form and personal information questionnaire. After collecting demographic information on age, gender, height, weight and familiarity of subjects with the method of tests, variables under study including balancing and falling risk indices were measured. It is worth noting that all subjects in this study were under the supervision of a neurologist and took medicine including Levodopa, Madopar, Trifen and Bromocriptine which was stable in dose and regularity of consumption and all tests were conducted at least 2 hours after taking these medicines.

\subsection{Participants and Exercise Protocol}

\subsubsection{Characteristics of the Experimental Group}

In the first exercise session, for the group taking Pilates exercises, basic information about Pilates exercise and the principles and method of taking exercise were provided by Pilates trainer to subjects. The exercise program was done three 1 h sessions per week for 8 weeks. Each session was began with 10 minutes of warm-up exercises, continued with 45 minutes of taking 10 selected Pilates exercise (standing side reach, bow and arrow, one leg stretch, oblique curl up, knee fold scissors, knee opening, shoulder bridge, diamond press, prone leg pull, torpedo) with the purpose of increasing the strength of body core muscles and lower limb joints' range of motion and finished with 5 minutes 
of cool down exercises. Before taking any exercise, its principles were explained to subjects, including the method of execution, keeping a desired body condition, trunk core muscles contraction and correct breathing during exercise. During exercises, there was adequate supervision for patients and in case of expression of any unpleasant and annoying feeling, including pain, dizziness, weakness or palpitation, continuation of exercises is forbidden. Exercises began with 6 - 8 replications and without any exercise resistance given the abilities of subjects, and in the process of implementing the protocol, the exercise volume (replication and duration) was regularly increased during 8 weeks considering characteristics of each subject and Pilates mini ball and Thera-Band were used for increasing the strength [19, 21, 22].

\subsubsection{Characteristics of the Control Group}

Subjects in the control group were similar to those of experimental one as much as possible and in addition to taking daily physical activities, they started walking in the duration similar to exercise group. Finally, after 8 weeks, the exercise protocol relating to balancing and falling risk was evaluated in both groups.

\subsection{Patient Assessment}

\subsubsection{Fullerton Advanced Balance Scale (FAB)}

Fullerton advanced balance scale (FAB) is a performance-based measure that comprehensively addresses the multiple dimensions of balance. The scale was specifically designed for use with independently functioning older adults. Performance on each of the 10 individual test items is scored using a 5-point ordinal scale (0 - 4) with a maximum score of 40 points possible. The total FAB score was used as a predictor variable in this study. The FAB scale is easy and quick to administer, can be conducted in a relatively small area, and requires approximately 10 to 12 minutes completing [23-25]. The test requires relatively inexpensive equipment to administer, including a stopwatch, pencil, 12-inch ruler, 6-inch-high bench (length, 18 in $(45.6 \mathrm{~cm})$; width, 14 in $(35.6 \mathrm{~cm})$; height, 6 in $(15.2 \mathrm{~cm}))$, masking tape, 2 foam pads (length, 18.5 in ( $47 \mathrm{~cm})$; width, 15 in; height, 2.5 in $(6.4 \mathrm{~cm})$ ), two 18 in lengths of nonslip material, a yardstick, and a metronome. Individual items on the FAB scale include static and dynamic balance activities performed in different sensory environments. The 10-item FAB scale involves the participant standing with feet together and eyes closed (item 1), reaching forward to retrieve an object (item 2), turning in a circle (item 3), stepping up and over a bench (item 4), tandem walking (item 5), standing on 1 leg (item 6), standing on foam with eyes closed (item 7), jumping for distance (item 8), walking with head turns (item 9), and recovering from an unexpected loss of balance (item 10). A full description of the scale and its associated test administration instructions is reported elsewhere [6, 7]. The FAB scale has shown high test-retest reliability $(0.96)$ as well as intra- $(0.92-1.00)$ and inter-rater reliability $(0.91-0.95)[23,24]$.

\subsubsection{Timed Up and Go Test (TUG)}

Timed Up and Go test (TUG) is a simple test used to assess a person's mobility and requires both static and dynamic balance. It uses the time that a person takes to rise from a chair, walk three meters, turn around, walk back to the chair, and sit down [26]. To perform the TUG, participants started sitting in a chair with a back. From this position, they were instructed on the word "Go" to stand up, walk out 3 meters from the chair, turn around, walk back to the chair and sit down as quickly as they could safely and without running. Performance was rated in seconds and participants were timed from the moment the rater said "Go" until they were seated again correctly in the chair with their back resting on the back of the chair. Previous research has established the reliability of this test as well as its sensitivity to medication states in persons with PD [26, 27].

\subsection{Data Analysis}

After data collection, in order to study normal data distribution, Kolmogorov-Smirnov test was used. Data on subjects' characteristics, including age, height, weight and other research variables were analyzed in two sections of descriptive and inferential statistics by SPSS software, version 22. For comparing the results obtained before and after the exercise intervention and also for comparing the results obtained in two groups, paired-samples T-test and independent-samples T-test were used respectively at the significance level of $\mathrm{P} \leq 0.001$.

\section{Results}

In this study, 30 patients with idiopathic Parkinson with disease level II-III in two experimental and control groups were investigated in terms of functional balance and falling risk.

In Table 1, the frequency distribution for each of the qualitative variables and descriptive characteristics of quantitative variables were explained in experimental and control groups.

The correlated T-test results in the experimental group showed that scores of functional balance $(\mathrm{P}=0.001)$ and falling risk $(P=0.001)$ were significantly improved after participating in the selected Pilates exercise program 
Table 1. Participants' Demographic Data $(\mathrm{N}=15)^{\mathrm{a}}$

\begin{tabular}{|c|c|c|}
\hline Variables & Experimental Group & Control Group \\
\hline Age & $57 \pm 6.24$ & $58.31 \pm 7.37$ \\
\hline \multicolumn{3}{|l|}{ Gender } \\
\hline Female & 7 & 5 \\
\hline Male & 8 & 10 \\
\hline Height, $\mathrm{cm}$ & $164.67 \pm 8.49$ & $164.94 \pm 8.83$ \\
\hline Weight, kg & $71.78 \pm 5.78$ & $73.40 \pm 8.50$ \\
\hline PD Duration, y & $7.27 \pm 3.80$ & $8.19 \pm 3.14$ \\
\hline \multicolumn{3}{|c|}{ H and Y Score (Range 1-5) } \\
\hline II & 5 & 6 \\
\hline III & 10 & 9 \\
\hline
\end{tabular}

while no significant difference was observed in scores of functional balance $(\mathrm{P}=0.364)$ and falling risk $(\mathrm{P}=0.816)$ (Table 2).

The results of dependent T-test showed a significant difference between the effects of the selected Pilates exercise program and walking on the functional balance and falling risk $(\mathrm{P}=0.001)$ (Table 3$)$.

\section{Discussion}

The present research was designed with the purpose of studying the effect of one period of the selected Pilates exercise program on the functional balance and falling risk among patients with Parkinson. Considering the results obtained in the present research, it was observed that taking the selected Pilates exercises for 8 weeks is accompanied by the significant improvement of functional balance in patients $(\mathrm{P}=0.001)$ while the difference observed in the functional balance of control group who walked in a similar duration was not significant $(\mathrm{P}=0.364)$. In addition, the difference evaluated between the score results of functional balance scale in the posttest was significant between two Pilates exercise and control groups $(\mathrm{P}=0.001)$. The postural instability was one of the most debilitating aspects of Parkinson disease and could lead to the increase of falling and reduction of mobility and functional capacity of patients. Patients with Parkinson suffer less stability range and more postural sways compared to their peers and the impaired postural control is significantly related to the increased falling risk in these people [3]. Many studies claim that taking regular exercises affects the improvement of motor and non-motor symptoms in these people. Indices of body posture control are important aspects affecting the independence maintenance and life quality among these patients who significantly look for improvable exercise interventions [5, 28, 29]. Different exercise interventions including aerobic exercises, hydrotherapy, balance exercises and walking could have significant treatment advantages with the increase of functional capacity among patients with Parkinson [7, 30]. There are different theories about the effect of exercise program on the improvement of balance [31]. Considering results of researches conducted, it was determined that exercises accompanied by resistance and balance exercises affected the improvement of balance disorders, especially in patients with mild and moderate levels of Parkinson more than other exercises [8]. Since Pilates exercises are a kind of resistance exercise in which the body weight is imposed and the principle of overload is in the form of replications increase and based on physiological principles, skeletal muscles can be severely influenced by these exercise [32]. The balance improvement resulted from the effect of Pilates exercises can be studied based on the theory of systems. According to the theory of systems, the ability of body control is the result of simultaneous and complex interaction between nervous, muscular and skeletal systems (postural control system). In order to control posture for maintaining balance and consequently motion through the above systems, the combination of sensory data (for determining the body condition in space) and the ability of musculoskeletal system for imposing proper force are necessary. In this model, the central nervous system is studied using the information of visual, vestibular and proprioceptive system (including joint position sense and environmental sense), the center of gravity's situation against the gravity and supporting surface conditions, and a proper motor response is provided in the form of pre-planned motor patterns. Therefore, referring to the theory of systems and the effect of physical activity on the improvement of each of these systems, Pilates exercises can be accompanied by the improvement of balance in patients with Parkinson who suffer balance disorders resulted from functional decline in the central nervous system $[33,34]$. Furthermore, the improvement of balance as the result of Pilates exercises is due to the improvement of muscular power and mental factors (stress and anxiety) in participants because the reduction of lower limb muscle strength puts the center of gravity in front of the ankle joint leading to a disturbance in balance and falling. On the other hand, improvement of muscular power can lead to the dislocation of center of gravity to the ankle and improve the balance. Therefore, it seems that Pilates exercise method affects the modification of impaired balance by focusing on the improvement of core muscular performance and body axial stability through muscular nervous 
Table 2. Differences Between Pretests and posttests in Experimental and Control Group for FAB and TUG

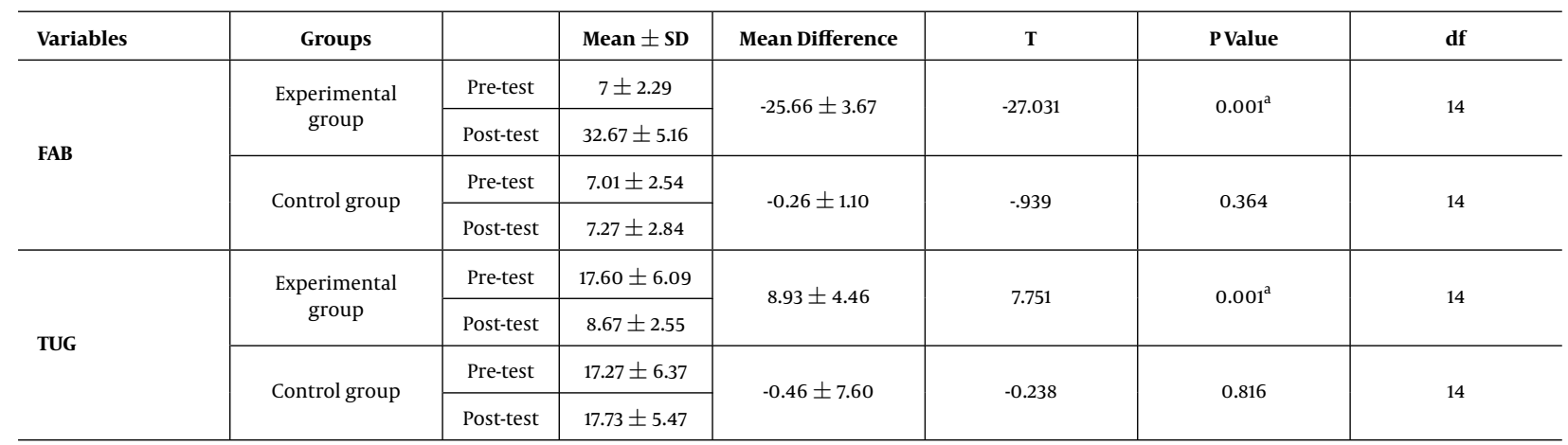

${ }^{\mathrm{a}}$ Significant difference at the significance level of $\mathrm{P} \leq 0.001$.

Table 3. Differences Between the Groups (Control vs. Experimental) for FAB and TUG

\begin{tabular}{lcrr}
\hline Variables & Mean Difference & T & PValue \\
\hline FAB & $25.40 \pm 1.52$ & 16.692 & $0.001^{\mathrm{a}}$ \\
TUG & $-0.96 \pm 1.55$ & -5.816 & 28 \\
\hline
\end{tabular}

${ }^{\mathrm{a}}$ Significant difference at the significance level of $\mathrm{P} \leq 0.001$.

compatibilities $[12,16,32,35]$. In this regard, according to Kibar et al. (2015), the effect of 8 weeks of Pilates exercises is accompanied by useful effects on the static and dynamic balance and body central endurance among elderly people while no significant relationship was found between the body central endurance and balance [36]. It was concluded by Kloubec (2010) that Pilates exercises were accompanied by a significant improvement in the body core muscular power in elderly people while they did not have any significant effect on the balance and body condition [37]. In the comparison of the effects of Pilate's exercises and balance exercises on the unstable level and balance abilities of elderly women, Hyun showed that both exercise methods significantly affected the improvement of static and dynamic balance among elderly women and were accompanied by the increase of balance abilities in these people. Nevertheless, considering the security of most Pilates exercises, it seems that they are a more reliable method for improving the balance capacity of elderly people [38]. Given a significant difference in the functional balance of people who participated in Pilates exercise program compared to the control group, it can be concluded that the selected Pilates exercise program used in this research has an optimal effectiveness in order to improve impaired balance among patients with Parkinson and results of the present research are consistent with results of Bird et al. (2013), Campos de Oliviera et al. (2015), Mesquita et al. (2015) and Jonson et al. (2013) [16, 18, 20, 33, 39].
In addition, results of the present research showed that taking the selected Pilates exercises for 8 weeks was accompanied by a significant improvement in the reduction of danger among patients with Parkinson $(\mathrm{P}=0.001)$ while no significant difference was observed in terms of falling risk in the control group $(\mathrm{P}=0.816)$ and the difference in results relating to the anticipation of falling risk in the posttest was significant between two groups $(\mathrm{P}=$ 0.001). Generally, patients with Parkinson are at risk two times more than other peers. In these patients, compensatory steps for keeping balance during falling were 1.5 times shorter than the normal state and they need an effort to achieve objects safely by keeping balance [40]. Many researches show that physical exercises lead to the improvement of control of situation and reduction of falling in patients with Parkinson [8, 31]. Research findings indicate positive effects of exercise interventions on the improvement of parameters relating to balance and walking as well as the reduction of falling among these patients. Results of studies conducted in this area show the early effectiveness of exercise on the improvement of balance components while there is no study on the early effectiveness of exercises on the reduction of falling in these people [11, 28]. In a research by Sparrow et al. (2016), the effect of progressive balance exercises and in a research by Allen et al. (2015), the effect of combined walking, balance and strength exercises on the reduction of falling, improvement of balance and reduction of fear to fall were significantly positive $[12,15]$. 
In this regard, in a research with the purpose of determining the possible effect of exercise program on the improvement of falling factors among patients with Parkinson, a significant improvement in factors such as dynamic balance, muscular power and walking quality among these people as the result of participating at strength and balance exercise programs was reported by Canning et al. (2009)[6]. Muscular weakness, reduction of ankle irritability and loss of muscle synergy mechanisms all have a role in the falling. Therefore, implementation of the physical readiness program is a proper strategy to avoid falling because it leads to the increase of muscular power, flexibility and motion control. By providing exercise opportunities and challenges for balance mechanisms, the physical activity improves them. In the meantime, Pilates is an exercise program which encourages the use of thinking for controlling muscles. It emphasizes the ability of muscles condition for balancing the body and supporting the improvement of vertebral column situation [29, 41]. Considering the significant difference in the reduction of falling risk in Pilates group compared to the control one, it can be concluded that one period of exercise program based on Pilates principles is accompanied by the reduction of falling risk among patients with Parkinson and the results obtained from this research are consistent with the results of studies conducted by Irez et al. (2011), Josephs et al. (2016) $[17,42-44]$. The effect of Pilates exercises on the reduction of falling risk through physical readiness factors including balance, muscular power and flexibility among elderly population was evaluated as a significant positive effect by Barker et al. (2016) and Bird et al. [22, 45]. Pilates exercise can lead to the improvement of balance and risk of factors affecting the falling of elderly people. On the other hand, some of the characteristics of a proper exercise program in reducing the falling risk among elderly people include strength during progressive exercises, specialty, proper intensity, implementation at home or in group and involvement of parameters affecting the body condition control, including Pilates exercise method.

\subsection{Conclusion}

Based on the fact that Pilates exercises involve both muscular and nervous systems of body, they can be useful in treating impaired balance, especially in groups with control of situation disorders. Therefore, this type of exercise program can be suggested as a modality for such patients.

\subsection{Limitations}

Due to the limitation in the selection of subjects, the present research was conducted on patients with Parkin- son at age group of 50 years old and both males and females were present. Furthermore, due to the limitation of patients' cooperation in the implementation of project, only patients with Parkinson levels II and III were selected as subjects. Since it was impossible to accurately control patients' medicine and or stop taking medicine during the research, subjects were entered into the study with the supervision of neurologists and with more similarity in terms of the intensity and duration of medicine.

\subsection{Suggestions}

It is suggested that in future studies with similar subjects, not only the increase of sample size and gender segregation of groups and duration of exercise interventions, but also the effect of other exercise methods on the patients' performance are studied in order to determine the most effective protocols for improving other disorders in any type of disease. In addition, researchers are suggested to use patients with disease levels III and IV in future studies.

\section{Acknowledgments}

Authors provide their appreciation and acknowledgement for University of Guilan deputy of research and technology, faculty of physical education and sports Sciences, University of Guilan, all neurologists in Rasht city and patients with Parkinson referring to neurology clinics in Rasht city who assisted researchers in conducting this research.

\section{Footnotes}

Authors' Contribution: Hassan Daneshmandi supervised the project. Babak Bakhshayesh provided the advisory supervision. Shaghayegh Sayyar performed the project, statistical analysis and prepared the first draft.

Financial Disclosure: This study was supported by department of physical education and sport Sciences, University of Guilan.

Ethical Considerations: This study was registered in the Iranian registry for clinical trial (IRCT) as IRCT2016071228885N1. They were briefed on the study aims and signed a written informed consent before enrollment. For safety purposes, the participants were instructed how to perform exercises safely with stable supports (such as a table) located nearby for additional support if required. 


\section{References}

1. Duncan RP, Earhart GM. Are the effects of community-based dance on Parkinson disease severity, balance, and functional mobility reduced with time? A 2-year prospective pilot study. J Altern Complement Med. 2014;20(10):757-63. doi: 10.1089/acm.2012.0774. [PubMed: 25192393].

2. Yitayeh A, Teshome A. The effectiveness of physiotherapy treatment on balance dysfunction and postural instability in persons with Parkinson's disease: a systematic review and meta-analysis. BMC Sports Sci Med Rehabil. 2016;8:17. doi: 10.1186/s13102-016-0042-0. [PubMed: 27274396]

3. Dona F, Aquino CC, Gazzola JM, Borges V, Silva SM, Gananca FF, et al. Changes in postural control in patients with Parkinson's disease: a posturographic study. Physiotherapy. 2016;102(3):272-9. doi: 10.1016/j.physio.2015.08.009. [PubMed: 26582134].

4. Ganesan M, Sathyaprabha TN, Gupta A, Pal PK. Effect of partial weightsupported treadmill gait training on balance in patients with Parkinson disease. PM R. 2014;6(1):22-33. doi: 10.1016/j.pmrj.2013.08.604. [PubMed: 24021298].

5. van der Kolk NM, King LA. Effects of exercise on mobility in people with Parkinson's disease. Mov Disord. 2013;28(11):1587-96. doi: 10.1002/mds.25658. [PubMed: 24132847].

6. Canning CG, Sherrington C, Lord SR, Fung VS, Close JC, Latt MD, et al. Exercise therapy for prevention of falls in people with Parkinson's disease: a protocol for a randomised controlled trial and economic evaluation. BMC Neurol. 2009;9:4. doi: 10.1186/1471-2377-9-4. [PubMed: 19161631].

7. Alves Da Rocha P, McClelland J, Morris ME. Complementary physical therapies for movement disorders in Parkinson's disease: a systematic review. Eur J Phys Rehabil Med. 2015;51(6):693-704. [PubMed: 26138090].

8. Corcos DM, Robichaud JA, David FJ, Leurgans SE, Vaillancourt DE, Poon C, et al. A two-year randomized controlled trial of progressive resistance exercise for Parkinson's disease. Mov Disord. 2013;28(9):1230-40. doi: 10.1002/mds.25380. [PubMed: 23536417].

9. Dibble LE, Addison O, Papa E. The effects of exercise on balance in persons with Parkinson's disease: a systematic review across the disability spectrum. J Neurol Phys Ther. 2009;33(1):14-26. doi: 10.1097/NPT.0b013e3181990fcc. [PubMed: 19265767].

10. Schlenstedt C, Paschen S, Kruse A, Raethjen J, Weisser B, Deuschl G. Resistance versus Balance Training to Improve Postural Control in Parkinson's Disease: A Randomized Rater Blinded Controlled Study. PLoS One. 2015;10(10):e0140584. doi: 10.1371/journal.pone.0140584. [PubMed: 26501562].

11. Shen X, Wong-Yu IS, Mak MK. Effects of Exercise on Falls, Balance, and Gait Ability in Parkinson's Disease: A Meta-analysis. Neurorehabil Neural Repair. 2016;30(6):512-27. doi: 10.1177/1545968315613447. [PubMed: 26493731].

12. Allen NE, Canning CG, Sherrington C, Lord SR, Latt MD, Close JC, et al. The effects of an exercise program on fall risk factors in people with Parkinson's disease: a randomized controlled trial. Mov Disord. 2010;25(9):1217-25. doi:10.1002/mds.23082. [PubMed: 20629134].

13. Li F, Harmer P, Fitzgerald K, Eckstrom E, Stock R, Galver J, et al. Tai chi and postural stability in patients with Parkinson's disease. $N$ Engl J Med. 2012;366(6):511-9. doi: 10.1056/NEJMoa1107911. [PubMed: 22316445].

14. Sedaghati P, Daneshmandi H, Karimi N, Barati AH. A Selective Corrective Exercise to Decrease Falling and Improve Functional Balance in Idiopathic Parkinson's Disease. Trauma Mon. 2016;21(1):e23573. doi: 10.5812/traumamon.23573. [PubMed: 27218051].

15. Sparrow D, DeAngelis TR, Hendron K, Thomas CA, Saint-Hilaire M, Ellis T. Highly Challenging Balance Program Reduces Fall Rate in Parkinson Disease. J Neurol Phys Ther. 2016;40(1):24-30. doi: 10.1097/NPT.0000000000000111. [PubMed: 26655100].

16. Bird ML, Hill KD, Fell JW. A randomized controlled study investigating static and dynamic balance in older adults after training with Pilates.
Arch Phys Med Rehabil. 2012;93(1):43-9. doi:10.1016/j.apmr.2011.08.005. [PubMed: 21975148].

17. Irez GB. The effects of different exercises on balance, fear and risk of falling among adults aged 65 and over. Anthropol. 2014;18(1):129-34.

18. Mesquita LS, de Carvalho FT, Freire LS, Neto OP, Zangaro RA. Effects of two exercise protocols on postural balance of elderly women: a randomized controlled trial. BMC Geriatr. 2015;15:61. doi: 10.1186/s12877015-0059-3. [PubMed: 26033080].

19. Barker AL, Talevski J, Bohensky MA, Brand CA, Cameron PA, Morello RT. Feasibility of Pilates exercise to decrease falls risk: a pilot randomized controlled trial in community-dwelling older people. Clin Rehabil. 2016;30(10):984-96. doi: 10.1177/0269215515606197. [PubMed: 26385357].

20. Campos de Oliveira L, Goncalves de Oliveira R, Pires-Oliveira DA. Effects of Pilates on muscle strength, postural balance and quality of life of older adults: a randomized, controlled, clinical trial.JPhys Ther Sci. 2015;27(3):871-6. doi: 10.1589/jpts.27.871. [PubMed: 25931749].

21. Canada PS . Exercise for people with parkinson's. Parkinson Society Canada. 2012

22. Bird ML, Fell J. Positive long-term effects of Pilates exercise on the aged-related decline in balance and strength in older, communitydwelling men and women. J Aging Phys Act. 2014;22(3):342-7. doi: 10.1123/japa.2013-0006. [PubMed: 23921206].

23. Hernandez D, Rose DJ. Predicting which older adults will or will not fall using the Fullerton Advanced Balance scale. Arch Phys Med Rehabil. 2008;89(12):2309-15. doi: 10.1016/j.apmr.2008.05.020. [PubMed: 18976981].

24. Rose DJ. Fallproof!: a comprehensive balance and mobility training program. Human kinetics; 2010.

25. Rose DJ, Lucchese N, Wiersma LD. Development of a multidimensional balance scale for use with functionally independent older adults. Arch Phys Med Rehabil. 2006;87(11):1478-85. doi: 10.1016/j.apmr.2006.07.263. [PubMed: 17084123].

26. Schoene D, Wu SM, Mikolaizak AS, Menant JC, Smith ST, Delbaere K, et al. Discriminative ability and predictive validity of the timed up and go test in identifying older people who fall: systematic review and meta-analysis. J Am Geriatr Soc. 2013;61(2):202-8. doi: 10.1111/jgs.12106. [PubMed: 23350947].

27. Foreman KB, Addison O, Kim HS, Dibble LE. Testing balance and fall risk in persons with Parkinson disease, an argument for ecologically valid testing. Parkinsonism Relat Disord. 2011;17(3):166-71. doi: 10.1016/j.parkreldis.2010.12.007. [PubMed: 21215674].

28. Canning CG, Paul SS, Nieuwboer A. Prevention of falls in Parkinson's disease: a review of fall risk factors and the role of physical interventions. Neurodegener Dis Manag. 2014;4(3):203-21. doi: 10.2217/nmt.14.22. [PubMed: 25095816].

29. Gao Q, Leung A, Yang Y, Wei Q, Guan M, Jia C, et al. Effects of Tai Chi on balance and fall prevention in Parkinson's disease: a randomized controlled trial. Clin Rehabil. 2014;28(8):748-53. doi: 10.1177/0269215514521044. [PubMed: 24519923].

30. Perez-de la Cruz S, Garcia Luengo AV, Lambeck J. Effects of an Ai Chi fall prevention programme for patients with Parkinson's disease. Neurologia. 2016;31(3):176-82. doi: 10.1016/j.nrl.2015.05.009. [PubMed: 26372407].

31. van Nimwegen $M$, Speelman $A D$, Overeem S, van de Warrenburg BP, Smulders K, Dontje ML, et al. Promotion of physical activity and fitness in sedentary patients with Parkinson's disease: randomised controlled trial. BMJ. 2013;346:f576. doi: 10.1136/bmj.f576. [PubMed: 23457213].

32. de Oliveira Francisco C, de Almeida Fagundes A, Gorges B. Effects of Pilates method in elderly people: Systematic review of randomized controlled trials. J Bodyw Mov Ther. 2015;19(3):500-8. doi: 10.1016/j.jbmt.2015.03.003. [PubMed: 26118523].

33. Johnson EG, Larsen A, Ozawa H, Wilson CA, Kennedy KL. The effects of Pilates-based exercise on dynamic balance in healthy adults. J Bodyw 
Mov Ther. 2007;11(3):238-42. doi: 10.1016/j.jbmt.2006.08.008.

34. Rueter LE, Jacobs BL. A microdialysis examination of serotonin release in the rat forebrain induced by behavioral/environmental manipulations. Brain Res. 1996;739(1-2):57-69. [PubMed: 8955925].

35. Irez BB. Pilates exercise positively affects balance, reaction time, muscle strength, number of falls and psychological parameters in 65+ years' old women. Middle East Technical University; 2009.

36. Kibar S, Yardimci FO, Evcik D, Ay S, Alhan A, Manco M, et al. Can a pilates exercise program be effective on balance, flexibility and muscle endurance? A randomized controlled trial. J Sports Med Phys Fitness. 2016;56(10):1139-46. [PubMed: 26473443].

37. Kloubec JA. Pilates for improvement of muscle endurance, flexibility, balance, and posture. J Strength Cond Res. 2010;24(3):661-7. doi: 10.1519/JSC.ob013e3181c277a6. [PubMed: 20145572].

38. Hyun J, Hwangbo K, Lee CW. The effects of pilates mat exercise on the balance ability of elderly females. J Phys Ther Sci. 2014;26(2):291-3. doi: 10.1589/jpts.26.291. [PubMed: 24648651].

39. Newell D, Shead V, Sloane L. Changes in gait and balance parameters in elderly subjects attending an 8-week supervised Pilates programme. J Bodyw Mov Ther. 2012;16(4):549-54. doi: 10.1016/j.jbmt.2012.02.002. [PubMed: 23036886].

40. Weaver TB, Robinovitch SN, Laing AC, Yang Y. Falls and Parkin- son's Disease: Evidence from Video Recordings of Actual Fall Events J Am Geriatr Soc. 2016;64(1):96-101. doi: 10.1111/jgs.13878. [PubMed: 26782857].

41. Caldwell K, Harrison M, Adams M, Triplett NT. Effect of Pilates and taiji quan training on self-efficacy, sleep quality, mood, and physical performance of college students. J Bodyw Mov Ther. 2009;13(2):155-63. doi: 10.1016/j.jbmt.2007.12.001. [PubMed: 19329051].

42. Irez GB, Ozdemir RA, Evin R, Irez SG, Korkusuz F. Integrating pilates exercise into an exercise program for 65+ year-old women to reduce falls. J Sports Sci Med. 2011;10(1):105-11. [PubMed: 24149302].

43. Josephs S, Pratt ML, Calk Meadows E, Thurmond S, Wagner A The effectiveness of Pilates on balance and falls in community dwelling older adults. I Bodyw Mov Ther. 2016;20(4):815-23. doi: 10.1016/j.jbmt.2016.02.003. [PubMed: 27814862].

44. Mehrholz J, Kugler J, Storch A, Pohl M, Hirsch K, Elsner B. Treadmill training for patients with Parkinson's disease. Cochrane Database Syst Rev. 2015(9):CD007830. doi: 10.1002/14651858.CD007830.pub4. [PubMed: 26363646].

45. Barker AL, Bird ML, Talevski J. Effect of pilates exercise for improving balance in older adults: a systematic review with meta-analysis. Arch Phys Med Rehabil. 2015;96(4):715-23. doi: 10.1016/j.apmr.2014.11.021. [PubMed: 25511371]. 